DOI: 10.25100/eg.v0i22.11402

Espacios y Territorios

\title{
Análisis geoespacial de actividades de reciclaje informal en la base de la pirámide social en Nezahualcóyotl, México ${ }^{1}$
}

\section{Geospatial analysis of informal recycling activities at the bottom of the pyramid in Nezahualcóyotl, Mexico}

\author{
Javier Gómez Maturano \\ Universidad Autónoma del Estado de México, Cuautitlán Izcalli, México. \\ jgomezma@uaemex.mx | 0000-0002-3385-3750 \\ Benito Sánchez Lara \\ Universidad Nacional Autónoma de México, Ciudad de México, México. \\ blara@unam.mx $\mid \underline{0000-0002-3385-3750}$
}

Para citar este artículo: Gómez Maturano, J. y Sánchez Lara, B. (2021). Análisis geoespacial de actividades de reciclaje informal en la base de la pirámide social en Nezahualcóyotl, México. Entorno Geográfico, (22), 123-143. https://doi.org/10.25100/eg.v0i22.11402

\section{Resumen}

En México los costos del manejo gubernamental de residuos son excesivos en un marco de gestión sostenible. La literatura reporta que las actividades económicas de adquisición de residuos en las grandes urbes revitalizan la economía local de zonas marginadas. El objetivo del trabajo fue analizar la distribución geoespacial de los centros de acopio privados [CAP] a partir de variables socioeconómicas que caracterizan a los sectores en base de la pirámide social en el municipio de Nezahualcóyotl. Este trabajo exploratorio utilizó la observación sistemática como estrategia de investigación principal: se realizó un análisis documental de variables sociodemográficas en bases de datos oficiales; con instrumentos de observación sistemática y recorridos virtuales se hizo la identificación y caracterización de CAP; se emplearon sistemas de información geográfica para analizar y representar la interacción de los CAP con su entorno social y económico. Los resultados permitieron caracterizar 293 CAP que se establecen en zonas de nivel socioeconómico bajo y con poca intensidad industrial. Los CAP constituyen fuentes de empleo para la base de la pirámide social dinamizando temporalmente la economía local. Los CAP hacen un uso intensivo del espacio público apropiándoselo para abatir los costos de operación lo que los hace insostenibles.

Palabras clave: Reciclaje, residuos sólidos urbanos, Economía informal, Análisis geoestadístico, Desarrollo sostenible, Sistema de información geográfica.

\footnotetext{
${ }^{1}$ Este artículo se deriva del proyecto de investigación doctoral del autor, titulado "Modelando un sistema de logística inversa para la gestión sostenible de residuos sólidos urbanos en la Ciudad de México", financiada por el Consejo Nacional de Ciencia y Tecnología, México, en el período de enero de 2017 a diciembre de 2020.
} 


\begin{abstract}
In Mexico, the costs of government waste management are excessive in a sustainable management framework. The literature reports that the economic activities of waste acquisition in large cities revitalize the local economy of marginalized areas. The objective of the work was to analyze the geospatial distribution of Private Collection Centers [PCC] based on socioeconomic variables that characterize the bottom of the pyramid in the municipality of Nezahualcóyotl. This is an exploratory work that used systematic observation as the main research strategy: a documentary analysis of sociodemographic variables was carried out in official databases; with systematic observation instruments and virtual tours, the identification and characterization of PCC was carried out; geographic information systems were used to analyze and represent the interaction of the PCC with their social and economic environment. The results made it possible to characterize 293 PCC that are established in areas of low socioeconomic status and with little industrial intensity. The CAP are sources of employment for the bottom of the pyramid, temporarily energizing the local economy. PCC make intensive use of public space, appropriating it to lower operating costs, which makes them unsustainable.

Keywords: Recycling, urban solid waste, Informal economy, Geostatistical analysis, Sustainable development, Geographic information system.
\end{abstract}

Recibido: 21 de agosto de 2020

Aceptado: 29 de abril de 2021

\title{
1. Introducción
}

Los residuos sólidos en México se clasifican en tres grandes grupos: residuos sólidos urbanos [RSU], residuos de manejo especial y residuos peligrosos (LGPGIRS, 2003). Los RSU son los residuos generados en las casas habitación, que resultan de la eliminación de los materiales que utilizan en sus actividades domésticas, de los productos que consumen y de sus envases, embalajes o empaques; los residuos que provienen de cualquier otra actividad dentro de establecimientos o en la vía pública que generen residuos con características domiciliarias, y los resultantes de la limpieza de las vías y lugares públicos, siempre que no sean considerados por esta Ley como residuos de otra índole (LGPGIRS, 2003).

Dado el incremento anual en la cantidad de RSU generados, tanto en México (SEMARNAT, 2016) como a nivel global (Global Reporting Initiative, 2015), y la pauperización de las condiciones de vida (Global Reporting Initiative, 2015), la recolección de materiales de los RSU ha proliferado en áreas urbanas.

La gestión de RSU para una ciudad debe incluir las actividades de generación, almacenamiento temporal, barrido, recolección, transferencia, transporte, tratamiento (reciclaje, composteo, incineración, tratamiento mecánico-biológico, etc.) y disposición final, buscando minimizar su generación, y maximizar el reciclaje (Morillas et al, 2017). Sin embargo, falta incentivar las actividades económicas de recuperación de RSU, su comercialización y mercados que los reintegren a las cadenas productivas; todo ello como alternativa para reducir los costos de gestión para las ciudades. Por ejemplo, la gestión de RSU tiene un costo para la Ciudad de México de 
alrededor de 157 millones de dólares al año (CIDAC, 2015), alrededor de 3,132 millones de pesos mexicanos.

La acepción más difundida, es aquella en la que el desarrollo sostenible es definido como el desarrollo que satisface las necesidades presentes sin comprometer la capacidad de las generaciones futuras de satisfacer sus propias necesidades (Cassen, 1987). Lamentablemente, la gestión de RSU en México sufre un fuerte atraso respecto al desarrollo que han alcanzado otros países. La normatividad en México asociada a los RSU habla de manejo integral y las actividades de manejo se concentran en la recolección y disposición final, principalmente en tiraderos a cielo abierto y en rellenos sanitarios. Lo mismo pasa en la Ciudad de México, que envía a disposición final, sin tratamiento previo, al 67.4\% de los RSU generados (SEDEMA, 2016). La gestión actual de RSU deja de lado los principios básicos de la gestión sostenible: minimización en la generación y maximización del reciclaje (Pires et al, 2011).

La iniciativa privada puede tener una oportunidad de negocio en las actividades de adquisición y reciclaje de RSU. Actualmente las actividades privadas de recolección, recuperación y comercialización de los subproductos de los RSU es un negocio de millones de pesos que ocupan a miles de personas; aunque la mayoría de los participantes son actores informales (Gómez Maturano, 2019).

Las redes de adquisición de residuos son actividades desarrolladas por personas consideradas en la base de la pirámide social, BOP, por sus siglas en inglés, the bottom of the pyramid. Se conoce como negocios BOP al concepto de administración de negocios con enfoque hacia los segmentos de población que han sido olvidados, grupos marginados por su bajo nivel de ingreso, y su integración en las cadenas de valor de los negocios (Bendul et al, 2017). Los mercados BOP incluyen un estimado de 2.7 a 4 mil millones de personas, con ingresos diarios promedio de 2 dólares (39.9 pesos mexicanos) o menos y un poder adquisitivo combinado de aproximadamente cinco billones de dólares (99 billones de pesos mexicanos) (Parmigiani y Rivera-Santos, 2015). Banco Interamericano de Desarrollo (2015) estima que más del 75\% de la población mexicana podría catalogarse como BOP, teniendo un mercado de 170 mil millones de dólares (3,392 mil millones de pesos mexicanos). La BOP en México es un mercado altamente atractivo para las corporaciones multinacionales por su acelerado crecimiento, porque las oportunidades son limitadas en los mercados desarrollados y debido a que la responsabilidad social se ha convertido en primordial (Parmigiani y Rivera-Santos, 2015).

Una forma de estratificarla a la población de un área urbana en México es a partir del Nivel Socio Económico [NSE], el cual constituye un nivel de bienestar del del hogar, asociado al nivel de ingreso y estilo de vida. El NSE es la norma desarrollada por Asociación Mexicana de agencias de Inteligencia de Mercado y Opinión (AMAI), basada en análisis estadístico, que permite hacer una segmentación social y mercadológica para analizar de una manera científica, estandarizada y sencilla, a los grupos que integran la sociedad mexicana. Actualmente la clasificación se conforma de siete segmentos o NSE: AB, C+, C, C-, D+, D y E. Los niveles D+, D y E son los NSE más bajos, los que representarían la población BOP en México. Estos niveles son explicados detalladamente en la sección de materiales y métodos.

Un Centro de Acopio Privado (CAP) es un establecimiento mercantil donde reciben y se acondicionan los materiales valorizables contenidos en los residuos sólidos urbanos y de manejo 
especial, o bien, donde se reciben, cuantifican, reúnen, trasvasan y acumulan temporalmente residuos para ser enviados a instalaciones autorizadas para su tratamiento, reciclaje, reutilización, coprocesamiento o disposición final (SEDEMA, 2016).

La aparición de los mercados BOP y la creciente importancia de las economías en desarrollo como nuevas fuentes de innovaciones frugales han atraído el interés de académicos y profesionales (Rosca et al, 2017). Estudios centrados en la recolección y adquisición de RSU son escasos, principalmente considerando su contribución social y económica. Un análisis general en este sentido lo presenta Rosca et al, (2017) al estudiar las innovaciones sostenibles para generar valor económico, medioambiental y social para mercados BOP. Estos autores identifican las innovaciones inversas, que son planteamientos frugales que generan nuevos modelos de negocio e incluyen estrategias de sostenibilidad. Los planteamientos frugales son aquellas ideas de negocio ahorrativas y económicas en el uso de recursos naturales, buscan optimizar el uso del tiempo y el dinero para evitar el desperdicio, el derroche o la extravagancia (Rosca et al, 2017).

Una de las propuestas de sostenibilidad en los negocios se puede identificar en Strandhagen et al, (2017) que proponen un modelo de negocio basado en cuatro elementos base: propuesta de valor, interfaz con el cliente, estructura de la cadena de suministro, modelo financiero.

En América Latina los esfuerzos son menores, pero se identifican los trabajos de Hincapie (2018) y Gutiérrez Gallego (2015). Hincapie (2018) evalúa la viabilidad de la exportación de RAEE (residuos de aparatos eléctricos y electrónicos) generados en los centros de acopio de población vulnerable en Colombia, con destino a la población de Guiyi en China; es destacable en este trabajo el enfoque social de las actividades de recolección y su carácter predominantemente informal. Gutiérrez Gallego (2015) desarrolla un modelo de negocio basado en la metodología Canvas para el aprovechamiento de los RAEE centrado la recolección y su posterior reciclaje.

Las actividades de reutilización y reciclaje de RSU constituyen una actividad economía significativa y su empleo asociado debe considerarse como un factor a considerar en las decisiones sociales relacionadas con la gestión de los RSU (Çetinsaya Özkir et al, 2015). La inclusión de variables sociodemográficas asociadas las actividades de reciclaje de RSU que se desarrollan en un área urbana particular; Nezahualcóyotl, en la zona centro de México, constituye la principal contribución de este trabajo.

El análisis geoespacial realizado en este trabajo incluye la recolección de datos sociodemográficos y económicos de la zona de estudio obtenidos de bases de datos del Instituto Nacional de Estadística, Geografía e informática [INEGI]. Dicha información fue refinada, procesada y visualizada mediante Sistemas de Información Geográfica [SIG], particularmente con el software QGIS. Con este análisis se logró establecer la correlación entre las actividades de los CAP y áreas urbanas con ciertas características sociodemográficas y económicas.

El análisis geoespacial y estadístico realizado en este trabajo, entre las actividades de reciclaje de RSU en CAP y entorno urbano no es común. Los SIG han sido utilizados para mejorar los sistemas de gestión de residuos sólidos principalmente en cuanto a la eficiencia del sistema (Sanjeevi y Shahabudeen, 2016). La revisión de la literatura hecha por Sanjeevi y Shahabudeen (2016) muestran que los trabajos de SIG aplicados en la gestión de residuos tienen como objetivo principal la mejora del desempeño técnico operativo del sistema, por ejemplo, al minimizar la distancia total de recolección, lo que ha reducido automáticamente el costo y el tiempo de recolección. 
Los problemas de ruteo de los vehículos de recolección de RSU en los países en desarrollo plantean una tarea difícil en la que los SIG han contribuido a simplificar. Apaydin y Gonullu (2007) estudiaron un área de sólo 40 km2 e identificaron el camino más corto para la recolección de RSU, utilizando SIG descubrieron que la distancia podría reducirse entre un 4 y 59\%, con las subsecuentes reducciones de costo (US \$ 0.07 a 0.21 por tonelada-km) y tiempo. Karadimas Nikolaos et al, (2007) utilizado ArcGIS Network Analyst y examinó un área de 1.34 km2 y solo una ruta en Atenas, Grecia y logró un ahorro del $20 \%$ en la longitud de la ruta.

Sin embargo, son pocos los estudios en los que se aplican SIG para modelar aspectos sociales o ambientales asociados a la gestión y valorización de RSU. El reciclaje informal ha sido practicado tradicionalmente por grupos marginales en los mercados emergentes, como Nezahualcóyotl, en la zona centro de México. Como parte de la exclusión social que sufren, las sociedades de recicladores generan sus propios hábitos y valores, y a menudo son objeto de acoso por parte de las autoridades y la policía (Wilson et al., 2006 citado en Fei, et al., 2016). Esto hace que los estudios de campo de estas actividades constituyen un reto para el investigador. En este trabajo se hace una caracterización operativa de los CAP en Nezahualcóyotl como negocios BOP y se establece su relación con el entorno urbano en el que se establecen: áreas urbanas con bajos niveles socioeconómicos y con poca actividad industrial. Dicho objetivo se logró gracias al uso de la observación sistemática de registros fotográficos y al uso de SIG.

\section{Materiales y Métodos}

Para contribuir a dar respuesta al planteamiento hecho en esta investigación se realizó un estudio exploratorio sobre los CAP que suministran materiales de los RSU a la industria del reciclaje en México. La base metodológica de este trabajo es la observación sistemática bajo la premisa de que para los fines de analizar las actividades de los CAP es mejor observar lo que realmente sucede en su operación: recopilación de datos en situaciones de la vida real, en el campo. La observación sistemática está vinculada con la producción de datos cuantitativos y el uso de análisis estadísticos (Denscombe, 2014).

A decir de Denscombe (2014) las ventajas de la observación sistemática son: 1) recogida directa de datos, registra directamente lo que hace el CAP, a diferencia de lo que podría decir su dueño o administrador; 2) es sistemático y riguroso, lo que requiere un programa de observación lo que proporciona una respuesta a los problemas asociados con la percepción selectiva de los observadores; 3 ) eficiente, pues proporciona un medio para recopilar cantidades sustanciales de datos en un período de tiempo relativamente corto; 4) datos precodificados, produce datos cuantitativos que están precodificados y listos para el análisis; 5) fiabilidad, pues genera confiabilidad en el observador, en el sentido de que dos o más observadores que usan un mismo cronograma deben registrar datos muy similares.

En la observación sistemática se requiere del campo a estudiar, lugares y sujetos, como de los aspectos concretos o conductas sobre las que se va a centrar la atención. Los elementos de la investigación son zona de estudio (lugar de la investigación), unidad de análisis (CAP) para estudiar los elementos de su operación y sostenibilidad BOP (objeto de estudio).

Estos contextos naturalistas o habituales (Anguera y Mendo, 2013) en que ha de observarse el comportamiento del fenómeno, lo constituye la zona de estudio seleccionada en esta investigación. 
El municipio de Nezahualcóyotl tiene una superficie de $51.436 \mathrm{~km}^{2}$. Fue seleccionado como zona de estudio para analizar los CAP, su localización se muestra en la Figura 1.

Fuente: Elaboración propia.

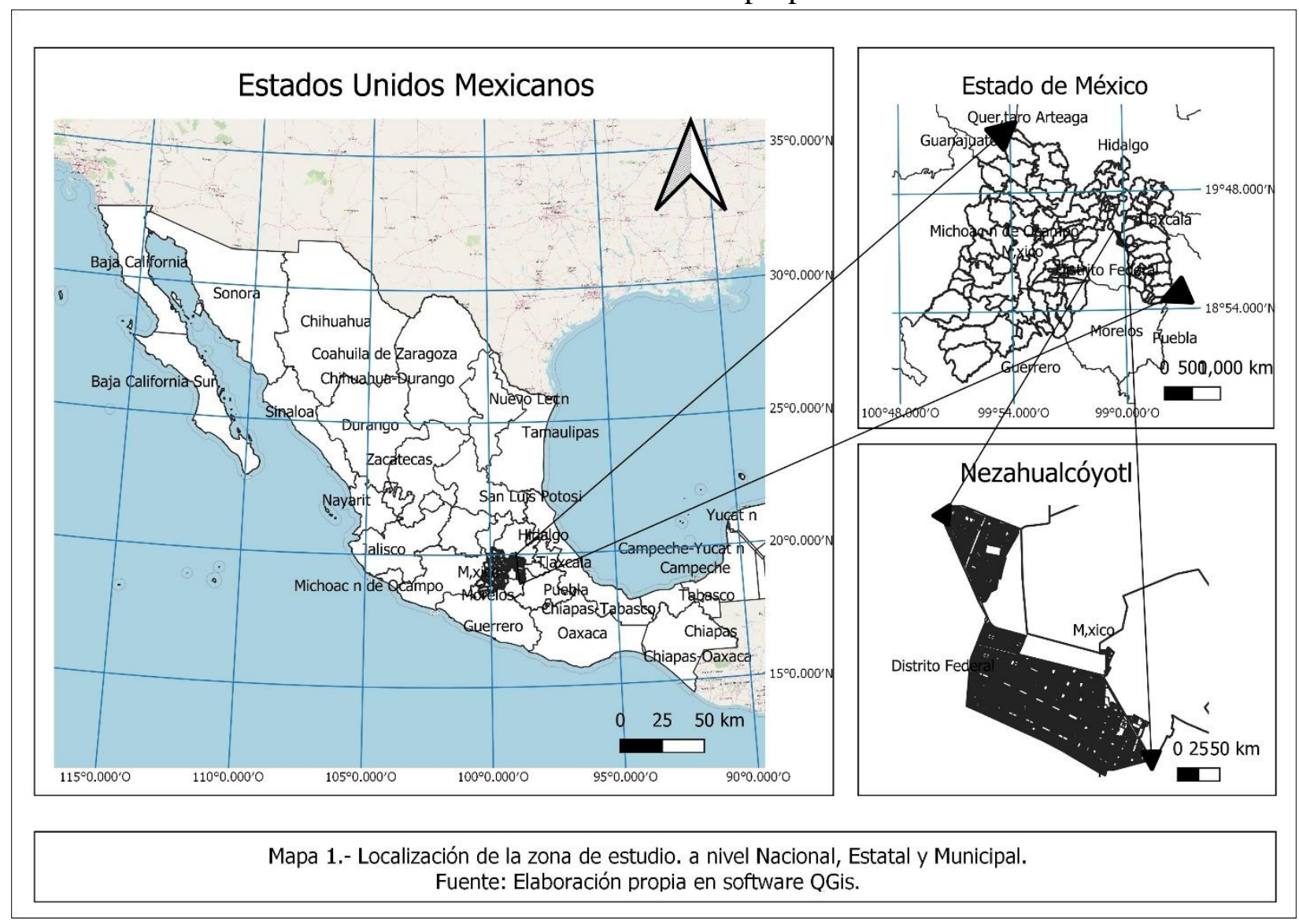

Figura 1. Localización de la zona de estudio a nivel Nacional, Estatal y Municipal.

Nezahualcóyotl se encuentra en la zona centro del país, que, en términos absolutos y relativos, es la zona que más RSU genera a nivel Nacional. Nezahualcóyotl era el municipio con mayor densidad poblacional en 2010, con 17,555.5 habitantes por $\mathrm{km}^{2}$. En ese año se recolectaban $820 \mathrm{t}$ de RSU diariamente y su tasa de recolección per cápita era de 0.746 kg/hab/día. Para 2016, la recolección diaria de RSU fue de 1200 t, con una tasa per cápita de 1.153 kg/hab/día. Aunque su tasa de recolección per cápita es menor a $2.0 \mathrm{~kg}$, Nezahualcóyotl es uno de los municipios que mayor incremento en la recolección de RSU ha tenido de 2010 a 2016, su crecimiento es del 46\%, lo que excede la media de crecimiento nacional en este rubro. A nivel nacional, este municipio ocupa el décimo lugar en el incremento en la recolección de RSU, en la ZMVM ocupa el tercer lugar, después de Ecatepec de Morelos y Benito Juárez. Dicha información fue obtenida de SIG por lo que constituyó su primer uso en este trabajo.

Debe señalarse que Ecatepec de Morelos, en el Estado de México, constituye también una zona de estudio adecuada, pero tiene una extensión 2.5 veces mayor a la del municipio de Nezahualcóyotl 
$\left(63.26 \mathrm{~km}^{2}\right)$, lo que, en términos operativos de la investigación, constituía un mayor consumo de recursos. Estos cálculos fueron obtenidos gracias al uso de SIG.

El análisis documental de bases de datos del INEGI y el uso de SIG permitió identificar la zona de estudio y caracterizar el entorno de la investigación. Las publicaciones gubernamentales y estadísticas oficiales como las del INEGI, son una propuesta ampliamente usada en la investigación social por ser una fuente de información documental con autoridad suficiente, objetiva y realista (Denscombe, 2014). Las ventajas de ocupar bases de datos documentales son (Denscombe, 2014): 1) permiten el acceso a grandes cantidades de información de forma relativamente fácil $y$ económica; 2) la investigación documental proporciona un método rentable para obtener datos, particularmente datos a gran escala, como los proporcionados por las estadísticas oficiales; 3 ) generalmente proporcionan una fuente de datos que es permanente y está disponible en una forma que otros pueden verificar.

En la Figura 2 se muestra el uso de SIG en la estrategia de investigación. El análisis espacial es uno de los usos fundamentales de los SIG, ya que permite generar nueva información de los datos existentes para orientar la toma de decisiones en áreas urbanas (Chang, 2016). Aunque la localización de los CAP en un mapa ya es en sí una forma de análisis espacial, los mapas de localización invitan a la interpretación de los patrones mostrados, esto es sólo un empleo básico que se le da al SIG en este trabajo. El análisis espacial aplica operaciones geográficas, estadísticas y matemáticas a los datos representados en el mapa (Chang, 2016).

Fuente: Elaboración propia.

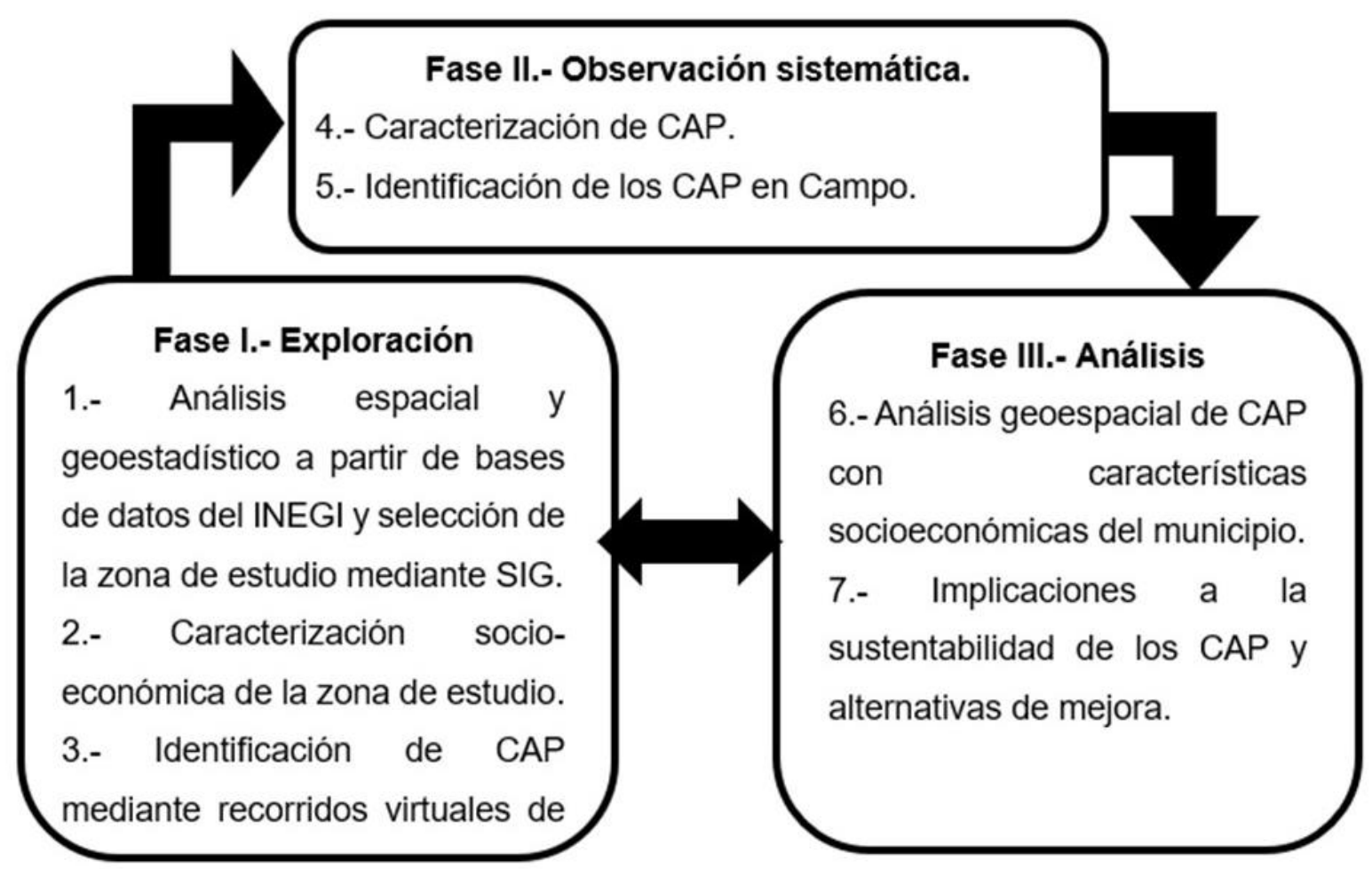

Figura 2. Estrategia de Investigación 
En este trabajo se utilizó SIG para generar estadística espacial y determinar la relación de contar un conjunto de puntos, localización de los CAP, con relación a variables sociodemográficas y económicas de los Áreas Geoestadísticas Básicas [AGEB]. El AGEB constituye la unidad básica del marco geoestadístico empleado por el INEGI, cuyo perímetro está representado generalmente por calles, avenidas, brechas y, en ocasiones, por rasgos físicos naturales y/o culturales, normalmente reconocibles y perdurables en el terreno (INEGI, 2010). El AGEB urbana se define como un área geográfica que se encuentra dentro de una localidad urbana (incluyendo todas las cabeceras municipales mayores o menores de 2500 habitantes); está integrada por un conjunto de manzanas edificadas y delimitadas por calles y avenidas, cuyo uso del suelo no es forestal ni agropecuario y que, partiendo de un punto, presenta continuidad física en todas direcciones o, en su caso, es interrumpida en forma notoria por terreno de uso no urbano (INEGI, 2010).

SIG es un campo de la tecnología que integra métodos y herramientas, para analizar y gestionar datos asociándolos a una referencia geográfica. SIG es un sistema informático para almacenar, administrar, analizar y mostrando datos geoespaciales ocupado en estudios sobre gestión de recursos naturales, planificación del uso del suelo, desastres naturales, transporte, atención médica, servicios públicos, análisis del área de mercado y planificación urbana (Chang, 2016). En este trabajo se ocupan SIG para hacer un análisis de las bases de datos del INEGI y elegir una zona de estudio, además para hacer una caracterización del entorno de los CAP. A partir de la exploración del Directorio Estadístico Nacional de Unidades Económicas [DENUE], que es un SIG, se determinó que Nezahualcóyotl es uno de los cinco municipios y alcaldías que mayor crecimiento ha tenido respecto del número de CAP que operan en ellos, tal como se explicó al inicio de esta sección. También fue necesario hacer el diseño de cuestionarios de observación, particularmente para la identificación de CAP y de observación de los elementos de CAP.

En el segundo punto de la Fase I, se realizó una caracterización socioeconómica de la zona de estudio. Esta caracterización se hizo a partir de los NSE propuesta por AMAI y que clasifica a la población en siete segmentos, mostrados en la Tabla 1.

Tabla 1. Definición de Niveles Socio Económicos.

\begin{tabular}{|l|l|}
\hline $\begin{array}{c}\text { Sivel } \\
\text { Ecio } \\
\text { Económico }\end{array}$ & \multicolumn{1}{c|}{ Características del hogar } \\
\hline AB & $\begin{array}{l}\text { Está conformado mayoritariamente (82\%) de hogares en los que el jefe de } \\
\text { familia tiene estudios profesionales. El 98\% de esos hogares cuenta con } \\
\text { Internet fijo en la vivienda. Es el nivel que más invierte en educación (13\% de } \\
\text { su gasto) y también el que menor proporción gasta en alimentos (25\%). }\end{array}$ \\
\hline C+ & $\begin{array}{l}\text { El 89\% de los hogares en este nivel cuentan con uno o más vehículos de } \\
\text { transporte y un 91\% tienen acceso a internet fijo en la vivienda. Un poco menos } \\
\text { de la tercera parte (31\%) de su gasto se destina a los alimentos y lo que se } \\
\text { destina (5\%) a calzado y vestido es muy homogéneo con otros niveles. }\end{array}$ \\
\hline $\mathbf{C}$ & $\begin{array}{l}\text { Un 81\% de los hogares en este nivel tienen un jefe de hogar con estudios } \\
\text { mayores a primaria y 73\% cuentan con conexión a Internet fijo en la vivienda. }\end{array}$ \\
\hline
\end{tabular}




\begin{tabular}{|l|l|}
\hline $\begin{array}{c}\text { Sivel } \\
\text { Ecio } \\
\text { Ecómico }\end{array}$ & $\begin{array}{c}\text { Características del hogar } \\
\text { Del total de gastos de este nivel, un 35\% son destinados a la alimentación y un } \\
9 \% \text { a educación. }\end{array}$ \\
\hline C- & $\begin{array}{l}\text { Un 73\% de los hogares en este nivel están encabezados por un jefe de hogar } \\
\text { con estudios mayores a primaria. El 47\% de estos hogares cuentan con } \\
\text { conexión a Internet fijo en la vivienda. El 38\% del gasto de estos hogares se } \\
\text { asigna para alimentos y un 5\% es para vestido y calzado. }\end{array}$ \\
\hline D+ & $\begin{array}{l}\text { En el 62\% de los hogares en este nivel el jefe de hogar tiene estudios mayores } \\
\text { a primaria. Solamente el 19\% cuenta con conexión a internet fijo en la } \\
\text { vivienda. Un 41\% de su gasto se destina a la alimentación y un 7\% a educación. }\end{array}$ \\
\hline D & $\begin{array}{l}\text { En el 56\% de hogares el jefe del hogar tiene estudios hasta primaria y } \\
\text { únicamente un 4\% tiene internet fijo en la vivienda. Un poco menos de la mitad } \\
\text { de su gasto (46\%) se destina a la alimentación. }\end{array}$ \\
\hline E & $\begin{array}{l}\text { La gran mayoría de hogares en este nivel (95\%) tienen un jefe de familia con } \\
\text { estudios no mayores a educación primaria. La posesión de internet fijo en la } \\
\text { vivienda es mínima (0.1\%). Es el nivel en el que la mayor parte de su gasto se } \\
\text { asigna a los alimentos (52\%) y el grupo en que se observa menor proporción } \\
\text { dedicada a la educación (5\%). }\end{array}$ \\
\hline
\end{tabular}

Fuente: Elaboración propia a partir de López Romo (2013).

Para estratificar el NSE de un hogar, debe aplicarse un cuestionario en el hogar para que a partir de ocho variables (ITDP México, 2016) se establezca el NSE. Para un nivel de mayor agregación, como el AGEB, existe una metodología para establecer el NSE predominante a partir de variables reportadas por INEGI. Esta metodología se reporte en ITDP México (2016). La metodología consiste en realizar un análisis por componentes principales a partir de tres índices socioeconómicos distintos, con el fin de obtener un solo puntaje que permite ordenar a las AGEB de acuerdo con el nivel socioeconómico predominante. Posteriormente, se le asigna un valor a partir de la distribución de la población de que pertenece a cada nivel socioeconómico calculada previamente por AMAI, utilizando la regla 8x7. Los índices presentados son:

$$
\begin{aligned}
\text { Índice computadora }= & \frac{\text { Número de viviendas con computadora en la } A G E B}{\text { Número de viviendas con alguno de los } 4 \text { bienes }} \\
& (\text { computadora, refrigerador, lavadora, televisión) }
\end{aligned}
$$

Índice escolaridad $=\frac{\text { Promedio de años estudiados en la AGEB }}{\text { Máximo del promedio de años estudiados de todas las AGEB }}$ 


$$
\text { Índice educación superior }=\frac{\begin{array}{c}
\text { Número de individuos mayores de } 25 \text { años } \\
\text { y con educación superior en la } A G E B
\end{array}}{\text { Número de individuos mayores de } 25 \text { años }}
$$

Los datos utilizados para calcular los índices provienen del Censo de Población y Vivienda de 2010 realizado por el Instituto Nacional de Estadística y Geografía (INEGI, 2010). Para ajustar la población a los NSE que calcula la AMAI, se recurrió a la estratificación nacional del año 2011: $6.8 \%$ de los hogares pertenecía al NSE AB, 14.2\% al NSE C+, 17\% al NSE C, $17.1 \%$ al NSE C-, $18.5 \%$ al NSE D+, 21.4\% al NSE D y 5\% al NSE E. Esta distribución población se ocupó para establecer los límites de cada NSE para el Índice 1, índice computadora, aplicado a los municipios del Estado de México con la información de INEGI (2010). De esta manera se pudo determinar el NSE predominante en cada uno de los 179 AGEB del municipio de Nezahualcóyotl, mismos que se muestran en la Figura 1.

Por otro lado, para hacer la caracterización de las AGEB en el municipio, por intensidad de actividades industriales, se ocupó el Sistema de Clasificación Industrial de América del Norte (INEGI, 2018), en el que se basa el DENUE. El DENUE es el registro nacional de millones de Unidades Económicas [UE], derivado de los Censos Económicos 2009, 2014 y 2019, y que se actualiza periódicamente y se enriquece con otras fuentes (INEGI, 2019). La clasificación de AGEB por intensidad industrial se realizó usando un conteo de puntos en SIG que estaban dentro de un polígono; particularmente los puntos representaban las UE, los polígonos fueron las AGEB del municipio. De esta manera, mediante SIG se contó el número de unidades económicas por AGEB. Posteriormente se aplicó el algoritmo de rupturas naturales de Jenks (1967). Este método calcula las diferencias de valores entre los individuos estadísticos ordenados de forma creciente y luego coloca un límite para separar los grupos donde las diferencias de valores son altas, de esta manera se hizo una categorización de AGEB por intensidad de actividades industriales. Los resultados de este procedimiento se muestran en la Figura 1.

La Fase II de la metodología tuvo como objetivo hacer un registro del número y localización de los CAP en la zona de estudio, haciendo recorridos virtuales por las calles del municipio con ayuda de la herramienta Google Maps ®. Para este propósito, las AGEB constituyeron la unidad mínima para de búsqueda de CAP. Para este objetivo, el municipio se zonifico, proponiendo un sistema con zonas pequeñas, el cual fue desagregado en AGEB posteriormente. El sistema zonal elaborado siguió las recomendaciones hechas en de Dios Ortúzar (2012), principalmente el de ser compatible con otras divisiones administrativas, particularmente con las zonas censales.

Una vez identificado un CAP en un recorrido virtual, se llenaba el cuestionario de observación sistemática. La información que se obtuvo con este cuestionario permitió la identificación y caracterización de CAP.

\section{Resultados}

A partir de la estrategia de investigación mostrada en la Figura 2, que está compuesta de tres fases, se obtuvieron los resultados que se presentan a continuación. La primera fase de la estrategia permitió hacer una exploración de las bases de datos del INEGI y un análisis geoespacial, con lo que se logró una caracterización socioeconómica de la zona de estudio. La segunda fase, de 132

Entorno Geográfico | № 22 | julio-diciembre 2021 | pp. 123-143 | https://doi.org/10.25100/eg.v0i22.11402 
observación sistemática, permitió la caracterización de los CAP y la identificación de impactos. La tercera fase, consistió en el análisis geoespacial de la distribución de los CAP y su relación con variables socioeconómicas de la zona de estudio.

\section{Caracterización socioeconómica de la zona de estudio}

El NSE constituye un nivel de bienestar del hogar, asociado al nivel de ingreso y estilo de vida. El NSE se ocupó en este trabajo para determinar la población que se encuentra BOP en la zona de estudio. Los AGEB con NSE más altos se encuentran en la zona norte de Nezahualcóyotl. Sólo quince AGEB tienen un NSE AB, en ellos habitaban un total de 79, 098 personas (7.1\% de la población del municipio en 2010). La superficie ocupada por estos AGEB representa el 10\% del territorio del municipio. La densidad poblacional de las AGEB con NSE AB es de 14, 412 habitantes por $\mathrm{km}^{2}$.

En contraste, la población con menor NSE en Nezahualcóyotl vive en condiciones críticas. Se identificaron cinco AGEB con NSE D. En estas AGEB vivían 18,568 personas. El nivel de hacinamiento en ellas era de 20, 217 habitantes por $\mathrm{km}^{2}$, una densidad poblacional $40 \%$ mayor a la observada en AGEB con NSE AB. Mientras que el número promedio de viviendas con vehículos particulares propios en las AGEB con NSE AB era 727 viviendas, en las AGEB con NSE D es de 249 viviendas (tres veces menor). El grado promedio de escolaridad en AGEB de NSE D era de 8.2 años, mientras que en AGEB con NSE AB era de 11.16.

En la Tabla 2 se muestran algunas características de las AGEB por NSE. La base de la pirámide social se compone de los NSE C-, D+ y D. Un total de 778, 202 personas pertenecían a alguno de estos NSE. Como puede apreciarse en la Tabla 2, a estos NSE les corresponde el menor grado de escolaridad y el menor porcentaje de viviendas con vehículo particular. Además de corresponderles la mayor cantidad de población a estos NSE, también se asocian a mayores niveles de hacinamiento en la zona de estudio.

Tabla 2. Características sociodemográficas de las AGEB según NSE predominante.

\begin{tabular}{|c|c|c|c|c|c|}
\hline NSE & AGEB & Población & $\begin{array}{l}\text { Densidad } \\
\text { poblacional } \\
\text { promedio } \\
{\left[\mathrm{Hab} / \mathrm{km}^{2}\right]}\end{array}$ & $\begin{array}{l}\text { Grado promedio } \\
\text { de escolaridad } \\
\text { [años] }\end{array}$ & $\begin{array}{l}\text { Porcentaje de } \\
\text { viviendas con } \\
\text { vehículo particular. }\end{array}$ \\
\hline AB & 15 & 79,098 & 14,412 & 11.16 & $56 \%$ \\
\hline $\mathrm{C}+$ & 9 & 50,823 & 17,780 & 11.47 & $48 \%$ \\
\hline $\mathbf{C}$ & 38 & 24,5834 & 22,532 & 9.7 & $36 \%$ \\
\hline $\mathrm{C}-$ & 39 & 265,275 & 22,554 & 9.28 & $33 \%$ \\
\hline D+ & 72 & 494,359 & 23,240 & 9.1 & $31 \%$ \\
\hline D & 5 & 18,568 & 20,217 & 8.2 & $26 \%$ \\
\hline $\mathbf{E}$ & \multicolumn{5}{|c|}{ Nivel socioeconómico no identificado en la zona de estudio. } \\
\hline
\end{tabular}

Fuente: Elaboración propia.

En la Figura 3 se muestra la distribución espacial de los NSE predominantes en las AGEB de Nezahualcóyotl. Como se mencionó al inicio de la sección, los NSE más altos se encuentran en la 
zona norte del municipio. Los NSE más bajos, se encuentran principalmente en los linderos de la zona de estudio, al suroriente del municipio, en los linderos con los municipios de Chimalhuacán y la Paz.

Fuente: Elaboración propia a partir de datos de INEGI (2010, 2018 y 2019).

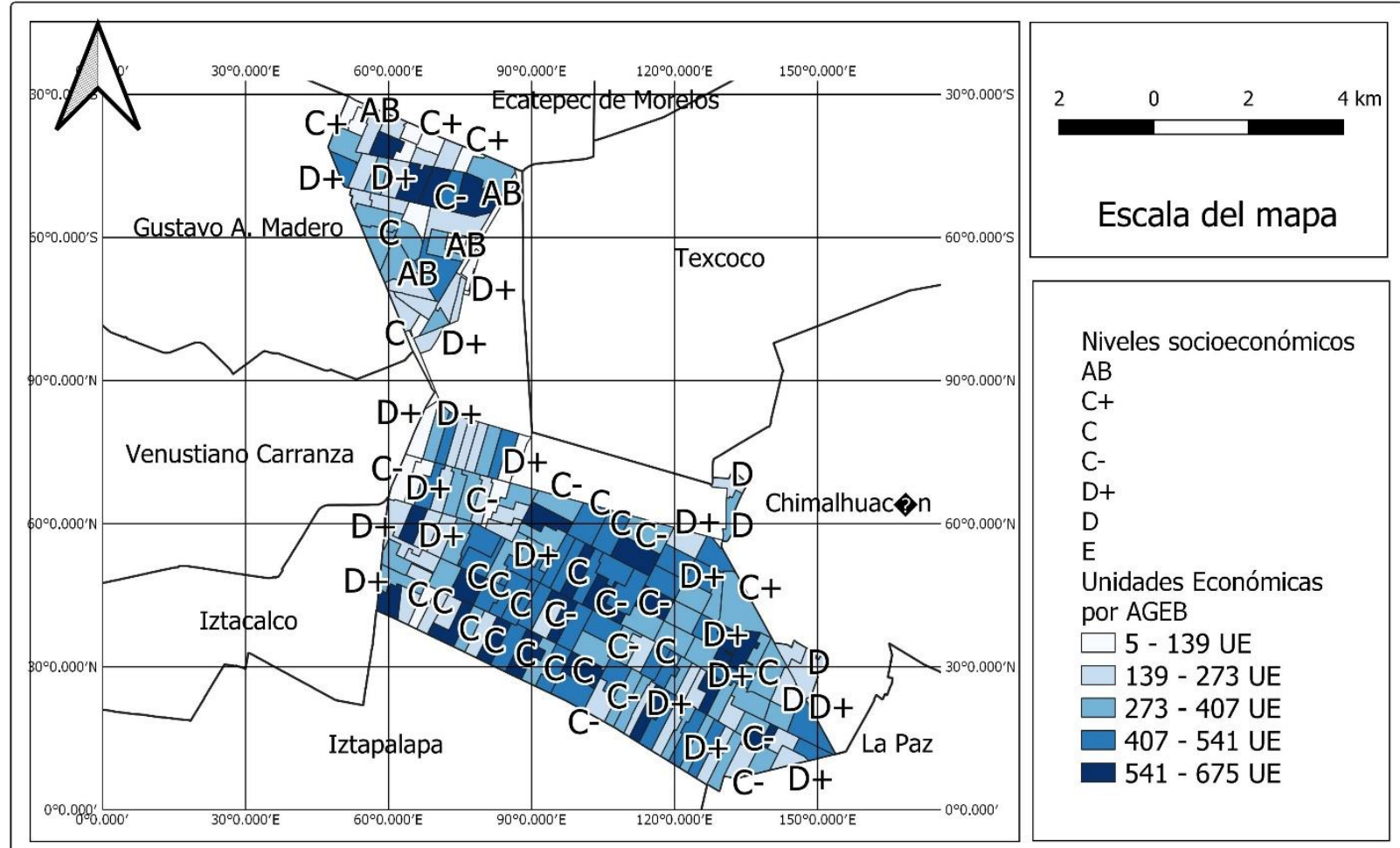

Mapa 2.- NSE predominante e intensidad de actividades económicas en las AGEB de Nezahualcóyolt, México. Fuente: Elaboración propia en software QGis con datos de INEGI (2010, 2015 y 2019).

Figura 3. NSE predominante e intensidad de actividades económicas en la EGEB de Nezahuakcóyolt, México.

La Figura 3 también muestra la intensidad de las actividades industriales en Nezahualcóyotl. Para determinar esta intensidad se ocupó el DENUE. En 2019 estaban establecidas 49, 166 UE en Nezahualcóyotl (INEGI, 2019). La mayor actividad industrial en la zona es el subsector 46, comercio al por menor, al que le corresponden el $47.3 \%$ de las UE. Le siguen el subsector 81, otros servicios, con el $17 \%$ de la actividad económica y el subsector 72 , servicios de alojamiento y preparación de alimentos, con el 10.5\%. La actividad industrial manufacturera en el municipio, subsectores 31-33, constituye apenas el $8.4 \%$ de las UE. El $91.61 \%$ de las UE tienen entre 0 y 5 trabajadores, lo que los constituye como microempresas.

En la Figura 3 se muestra la intensidad de la actividad industrial en el municipio de Nezahualcóyotl. Se consideró para esta clasificación establecer cinco categorías de intensidad en las actividades industriales, mismas que pueden verse en la Figura 3. Las AGEB con muy baja actividad industrial tienen de 5 a 139 UE, representan menos del 12\% del total de AGEB. El 26\% de AGEB tienen baja actividad industrial, es decir que tienen entre 139 y 273 UE ver Figura 4. El 28\% de las AGEB se categorizaron como intensidad industrial media, tiene más de 273 UE y hasta 407. Las AGEB 134

Entorno Geográfico | $\mathbf{N}^{\circ} 22$ | julio-diciembre 2021 | pp. 123-143 | https://doi.org/10.25100/eg.v0i22.11402 
intensidad industrial alta, de 407 a $541 \mathrm{UE}$, representan el 26\%. El resto de AGEB fueron categorizadas con intensidad industrial muy alta, el 8\%. En la Figura 4 se muestra detalladamente la dispersión de AGEB conforme al número de UE establecidas en ellas en 2019.

Fuente: Elaboración propia a partir de datos de INEGI (2019).

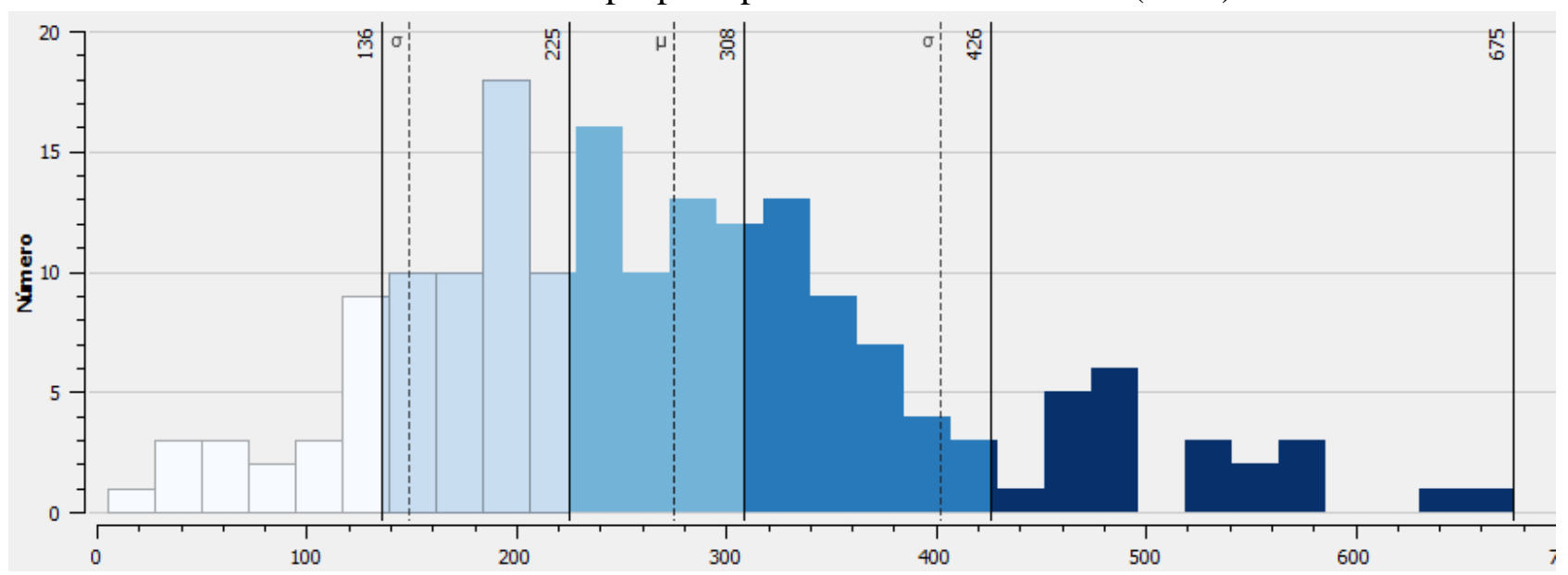

Figura 4. Categorización de AGEB por intensidad industrial en Nezahualcóyotl.

\section{Caracterización de centros de acopio privados}

Se identificaron un total de 293 CAP mediante los recorridos virtuales, identificados entre el 28 de febrero y el 1 de mayo de 2019 mediante observación sistemática. La antigüedad de las fotografías registradas en Google Maps ® es un elemento que limita la utilización de los datos encontrados para hacer inferencias respecto a la intensidad de los CAP en Nezahualcóyotl.

Respecto al giro de los CAP identificados, el 95\% se concentra en el acopio, compra y venta de RSU valorizables. Sólo el 5\% se concentra en el desmantelamiento, reparación, restauración o remanufactura de los residuos adquiridos. En la Figura 5 se muestra la localización de los 293 CAP identificados en Nezahualcóyotl. También de muestra el tipo de residuo en el que se concentran principalmente los CAP. Debe aclararse que el $71 \%$ de los CAP identificados acopian más de un residuo; por tanto, la clasificación presentada en la Figura 5 toma en cuenta el residuo principal que se observó en el CAP no implica que sea el único residuo que se acopia en dicho CAP. Sólo 85 de los 293 CAP identificados se especializan en un único tipo de residuo.

Los tres tipos de residuos más acopiados en los CAP de Nezahualcóyotl son: el papel, cartón y productos de papel, acopiados en el 58\% de los CAP; plásticos, principalmente PET, presente en el mismo porcentaje de CAP; los residuos metálicos, que incluyen los metales ferrosos, no ferrosos y el aluminio, se acopia en el 53\% de los CAP. Estos tres residuos constituyen la simbología empleada en la Figura 5. Sólo 85 CAP se concentran en un residuo, 30 se concentran en el manejo de plástico, 25 en el manejo de papel, cartón y otros derivados del papel.

El $60 \%$ de los CAP identificados tienen un establecimiento fijo para el desarrollo de sus actividades, $10 \%$ cuentan con establecimientos semifijos y $30 \%$ realizan las actividades de acopio en el interior de la vivienda. 
Fuente: Elaboración Propia.

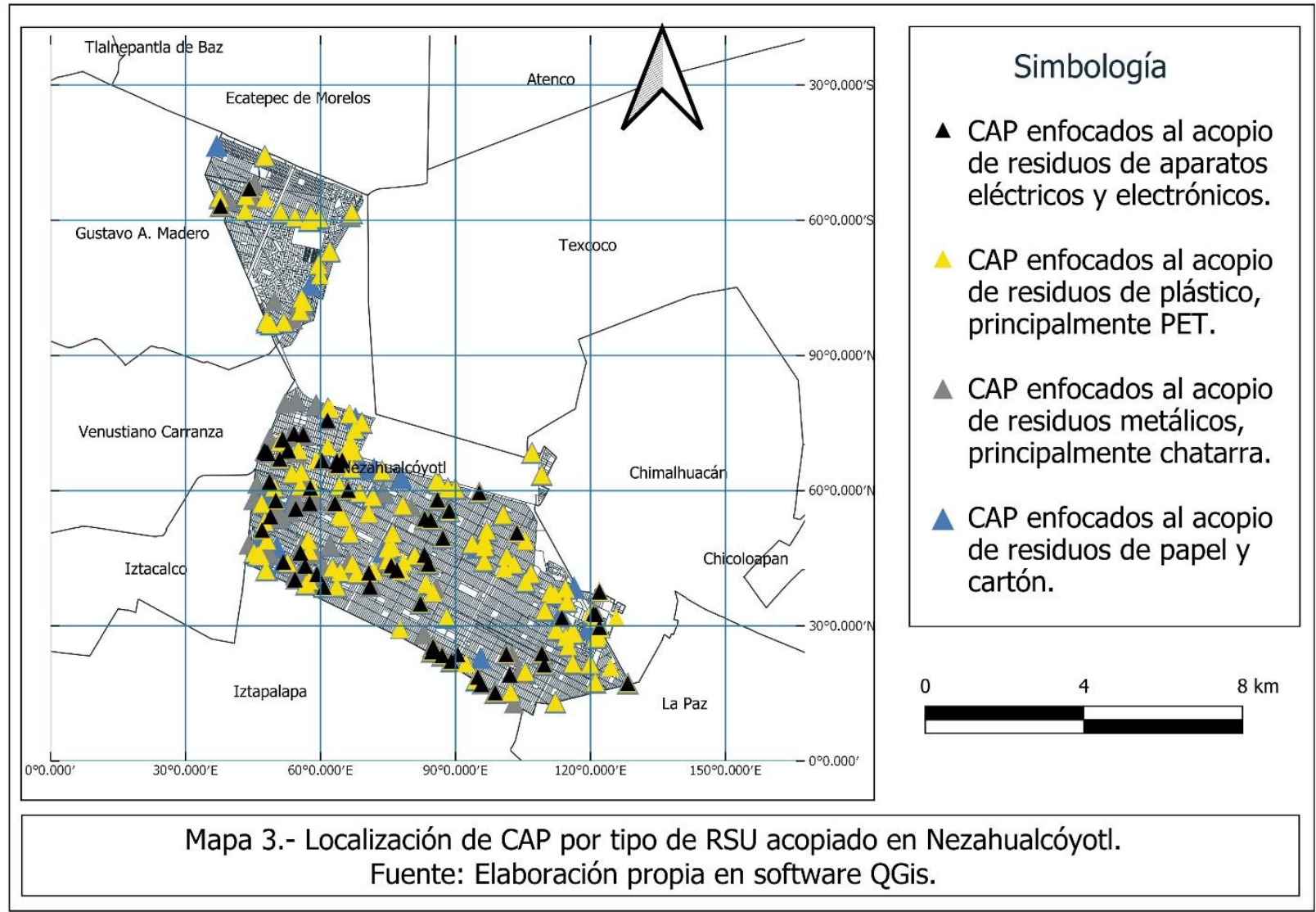

Figura 5. Localización CAP por tipo de RSU acopiado en Nezahualcóyotl.

\section{Análisis geoespacial de CAP en Nezahualcóyotl}

La localización de los CAP se muestra en la Figura 5, en esta localización debe observarse que la distribución de los CAP en la zona de estudio no es homogénea ni aleatoria. El hallazgo principal en la etapa de identificación y localización de CAP es que los CAP se aglutinan en AGEB focalizadas, con características sociales y económicas bien definidas. En promedio, en el municipio hay 3.2 CAP por AGEB. Sin embargo, en la Figura 5 pueden identificarse zonas en el interior de Nezahualcóyotl en las que no hay CAP, mientras que algunas AGEB llegan a tener hasta ocho CAP.

Una revisión de la sección izquierda de la Figura 6, que representa la zona norte del municipio, permite visualizar que en los NSE más altos prácticamente no hay CAP. El NSE predominante es determinante para que se establezca o no un CAP en la AGEB, más aún en los NSE más altos ( $\mathrm{AB}$ y $\mathrm{C}+$ ). Esto es claro en la sección izquierda de la Figura 6, pues en ninguna de las doce AGEB de NSE AB se ubican CAP. Sólo 1.3\% de los CAP identificados estaban establecidos en AGEB con NSE AB, los únicos cuatro CAP localizados en ellos se encontraban en los límites de las AGEB. En NSE C+ es el segundo nivel más alto en la clasificación poblacional, en las AGEB con este NSE predominante sólo se encontraron dos CAP. 
Fuente: Elaboración propia.

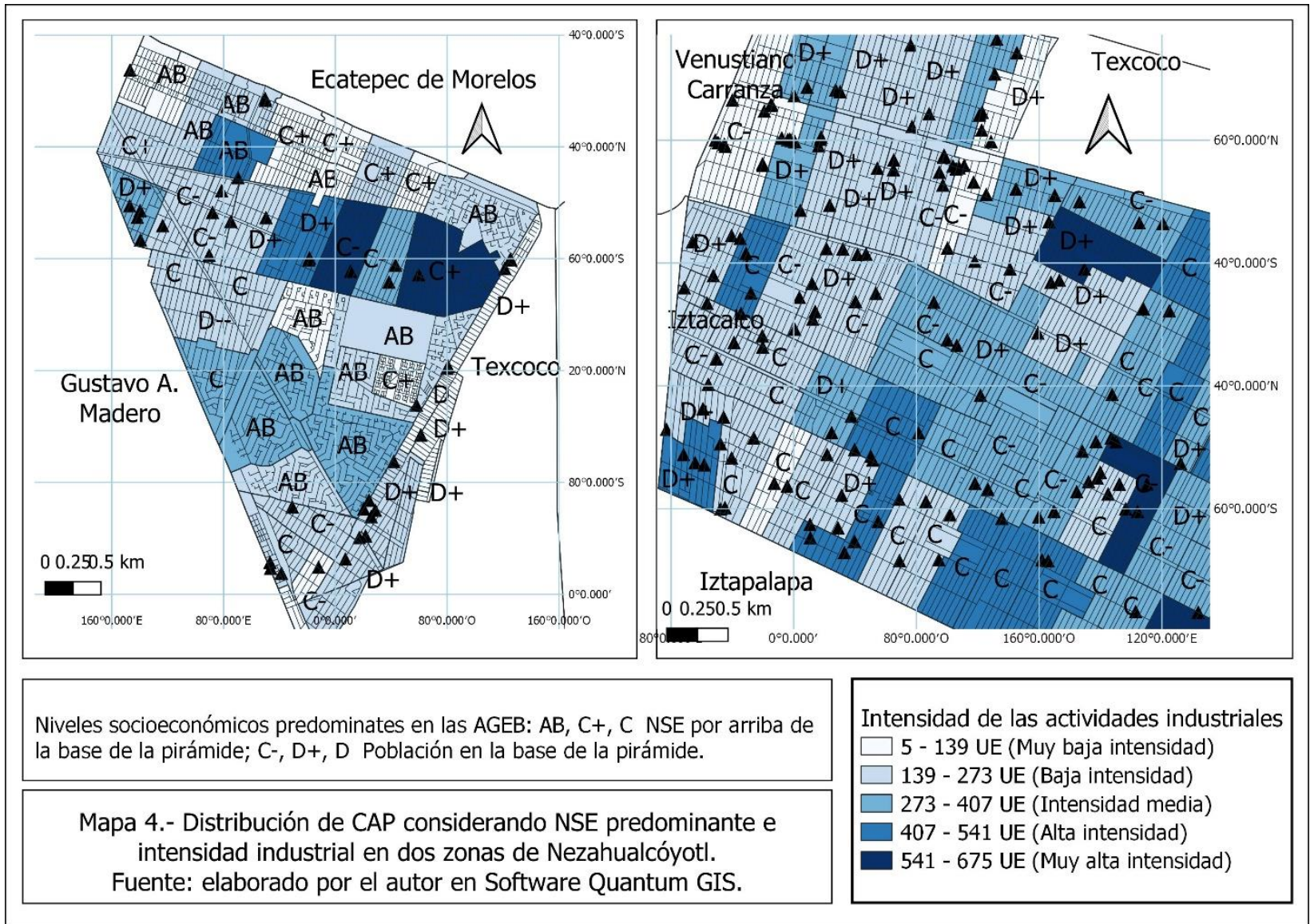

Figura 6. Distribución de CAP considerando NSE predominante e intensidad industrial en dos zonas de Nezahualcóyotl.

Es claro que a mayor NSE haya en la AGEB menor será la cantidad de CAP establecidos en ellas. La zona norte de Nezahualcóyotl, sección izquierda de la Figura 6, muestra como los NSE AB y C+ están libres de CAP. En esta zona de Nezahualcóyotl, los NSE AB y C+ se concentran en la parte central y en el extremo norte ver sección izquierda de la Figura 6 y se puede corroborar que no hay presencia de CAP. Sin embargo, en las AGEB contiguas, que circulan literalmente a estos centros de bienestar económico y social, se encuentran habitadas por personas con menor NSE. En estas AGEB con menor NSE si se ubican un número considerable de CAP. En la sección izquierda de la Figura 6 puede verse un cinturón de CAP que muy seguramente se concentra en el aprovechamiento de los RSU generados en las AGEB de mayor NSE. El cinturón de AGEB marginales con población BOP, NSE C-, D+ y D tiene un total de 40 CAP.

Los NSE más bajos registrados en el municipio, C-, D+ y D, son los AGEB que concentran la mayoría de los CAP, pero es destacable que el estrato más bajo, el NSE D sólo tiene 14 CAP. El $46.52 \%$ de los CAP del municipio se encuentran establecidos en AGEB con NSE predominante D+. Como puede verse en las dos secciones de la Figura 6, la ubicación de los CAP está relacionada al NSE del AGEB, a menor NSE es más común encontrar más CAP y a mayor NSE se esperaría 
no encontrase CAP, o encontrarlos en menor cantidad. Esta relación puede verse claramente en la Figura 7.

Sin embargo, la ubicación de CAP en AGEB específicas no se explica sólo por el NSE de estas. La intensidad de las actividades industriales también influye en el establecimiento o no de CAP. Esta intensidad se muestra con un relleno degradado en las dos secciones de la Figura 6. En este mapa el azul más oscuro representa AGEB con muy alta intensidad industrial y conforme el tono de azul se hace más claro, la intensidad de las actividades industriales baja. Una inspección en de la Figura 6 puede llevar al lector a observar que en las AGEB con mayor intensidad de actividades industriales el número de CAP es reducido, en comparación con aquellas AGEB de intensidad industrial media y baja.

Los datos mostrados en las de la Figura 7 y 8 muestran una fuerte correlación entre localización de CAP y NSE del AGEB, así como correlación con la intensidad industrial y la localización de CAP. 38 CAP estaban establecidos en AGEB con menos de 139 UE, 13.01\%. 129 CAP se encontraban establecidos en AGEB con entre 139 y 273 UE, lo que representa un 44.48\%. 80 CAP identificados en los recorridos virtuales estaban establecidos en AGEB con entre 273 y 407 UE, 27\% del total. 32 CAP se establecieron en AGEB que tenían más de 407 UE y menos de 541, un 10\%. Sólo 13 CAP se establecieron en los AGEB en los que hay mayor actividad económica.

Fuente: Elaboración propia.

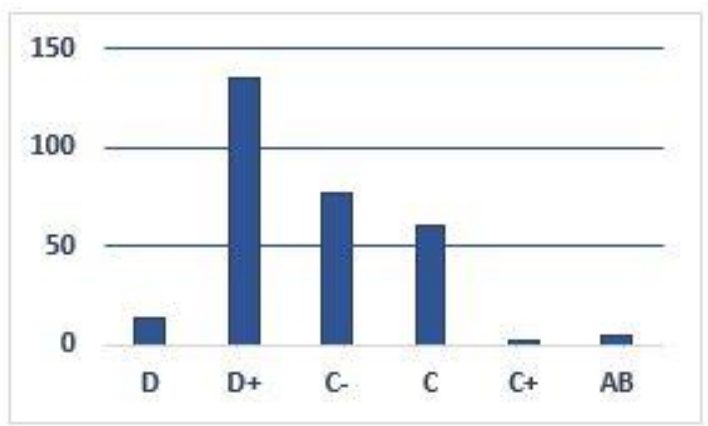

Figura 7. CAP en cada NSE predominante por AGEB.

Fuente: Elaboración propia.

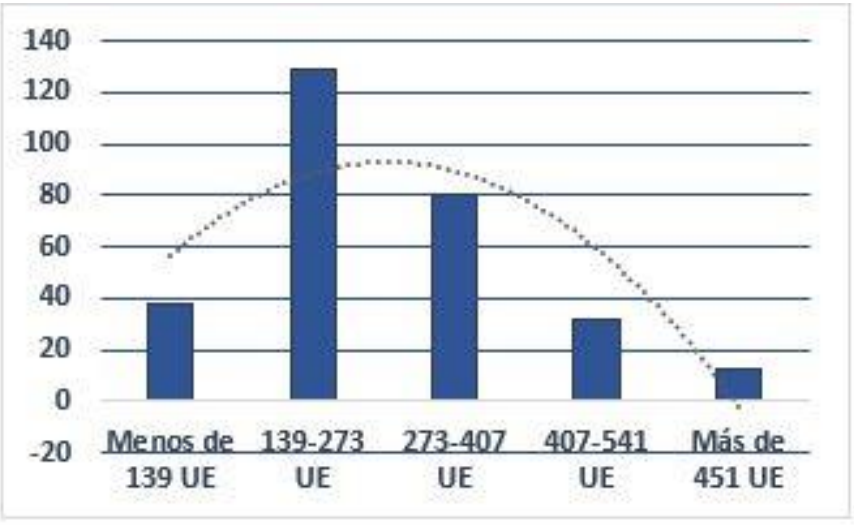

Figura 8. CAP según intensidad industrial por AGEB. 


\section{Implicaciones a la Sostenibilidad de los CAP y alternativas de mejora}

Espacialmente los CAP se localizan en los cordones de las áreas de mayor NSE: los CAP raramente se establecen en áreas geográficas con altos niveles socioeconómicos en su población o con intensa actividad económica. Esto es destacado, puesto que SEMARNAT (2016) y Horvath et al, (2018) señalan que, a mayor nivel de ingreso de la población, mayor será la generación de RSU y por tanto mayor la disponibilidad de residuos para acopiar y comercializar en CAP. Sin embargo, no es en las zonas de mayor generación de RSU, AGEB con mayor NSE y mayor intensidad en actividades industriales, en las que se establecen más CAP.

Los CAP se establecen en áreas en las que la población tiene un bajo nivel socioeconómico y dónde la actividad económica es escasa. Todos ellos ocupan la vía pública para el desarrollo de sus actividades, ocupan en promedio $150 \mathrm{~m}^{2}$ de espacio público para realizar sus operaciones, lo que constituye una superficie agregada de $43,950 \mathrm{~m}^{2}$ en el municipio. El almacenamiento de los residuos al aire libre y en vía pública, contribuye al deterioro de la calidad del aire, tanto localmente como en los alrededores, a causa del polvo que levanta el viento en los períodos secos. El almacenamiento de residuos al aire libre provoca que se transporte a otros lugares microorganismos nocivos que producen infecciones respiratorias, irritaciones nasales y de los ojos, a esto le sumamos las molestias que dan los malos olores (Escalona Guerra, 2014). En temporada de lluvias el almacenamiento de residuos puede ocasionar el filtrado de contaminantes al subsuelo por efecto de escurrimiento (Escalona Guerra, 2014). Estás áreas de almacenamiento se ocupan permanentemente constituyendo una fuente idónea para los vectores de enfermedades a través de fauna nociva.

\section{Discusión}

En este trabajo se ha mostrado la distribución espacial de los CAP en la zona de estudio y su posible relación con actividades económicas y los niveles socioeconómicos de la población. Este es un primer acercamiento al conocimiento del sistema de CAP que se encarga del reciclaje y valorización de RSU en un área urbana, ocupando SIG y herramientas de observación virtual.

Como señala Farahbakhsh y Forghani (2019), el estudio de los problemas económicos, la contaminación nociva y la degradación medioambiental producida por la gestión de RSU en zonas urbanas, es un problema para resolver a nivel mundial. Sin embargo, como señalan Çetinsaya Özkir et al, (2015) las condiciones de marginación económica y exclusión social en que se desarrollan estas actividades hacen difícil su estudio, principalmente en países emergentes. En este trabajo se ha mostrado que el uso de SIG y herramientas de observación virtuales puede acercar a los investigadores al fenómeno de la recolección y reciclaje informal.

Pese a que el marco normativo establece que la gestión de los RSU es responsabilidad de los Gobiernos Municipales (LGPGIRS, 2003), la participación privada en las actividades de acopio en la zona de estudio es alta. Doscientos noventa y tres agentes acopiadores fueron identificados en la zona de estudio, este número de CAP es mayor al número de centros de acopio públicos establecidos en toda la Zona Metropolitana del Valle de México (INEGI, 2019). La identificación de CAP por medio de recorridos virtuales permitió obtener la localización de los nodos de una red informal (Ver Figura 5) de acopio y reciclaje de RSU que funciona al margen del sistema público de gestión de RSU. 
Particularmente, en este trabajo se han identificado áreas urbanas con características específicas en las que operan CAP: bajo NSE y baja intensidad industrial. En estas AGEB concretas se puede presentar mayor contaminación y degradación medioambiental, producto de las actividades de los CAP. Estas afectaciones, se concentra principalmente en zonas con población BOP. Definir incentivos legales, económicos y sociales para apoyar el reciclaje, como lo proponen Farahbakhsh y Forghani (2019) podría mejorar el desempeño de los CAP. La reducción de los impactos medioambientales y sociales de los CAP es necesaria en estás AGEB, pues en ellas se establece el $60 \%$ de la población de la zona de estudio.

Estas redes de CAP son elementos nocivos inherentes al sistema de economía lineal en mercados emergentes. Como se muestra en este trabajo, las redes de CAP operan a gran escala predominantemente en la informalidad económica ocasionando graves problemas económicos, sociales y urbanos, incrementando la desigualdad social y espacial. Un desarrollo urbano desigual en las grandes urbes latinoamericanas en las últimas tres décadas muestra una ciudad fragmentada, donde zonas de bienestar generalizado generan grandes cantidades de RSU y son los cinturones de pobreza que los rodean quienes pepenando estos residuos recuperan materiales valorizables para comercializarlos. Este desarrollo fragmentado puede verse claramente en la zona norte del municipio de Nezahualcóyotl.

Grandes cantidades de RSU son desechados en estas zonas del bienestar con altos NSE, lo que da trabajo a los acopiadores informales que abastecen a los CAP conformándose así la red de adquisición establecida en la zona de estudio. Es la población de la base de la pirámide social la que dadas sus condiciones de exclusión y marginalidad aceptan la comercialización de materiales de bajo valor residual para tener un sustento. Los centros de acopio privados son un modelo de negocio de gran alcance en el municipio, particularmente del PET y residuos metálicos, así como cartón. Gracias a esta red de CAP es difícil encontrar botellas de PET y otros residuos valorizables en la calle, ilustrándose el impulso económico que tiene la cadena del reciclaje gracias a estos negocios.

El acopio de RSU valorizables en el municipio implica un esquema poco sostenible, con sus limitaciones, genera recursos económicos y empleos. Se está lejos de esquemas de manejo sostenible de los RSU, los impactos sociales y ambientales se concentran en la población BOP. El principal impacto identificado en este trabajo es la apropiación del espacio público para almacenar temporalmente los residuos en la vía pública. Esto es consistente con lo que señalan Çetinsaya Özkir et al, (2015), pues las actividades de reciclaje se hacen en la calle y se asocian con riesgos, entornos antihigiénicos, actividades delictivas, falta de vivienda, desempleo, pobreza y atraso, además son excluidos de la gestión gubernamental de RSU, tal como fue observado en Nezahualcóyotl.

\section{Conclusiones}

En este trabajo se recurrió a la observación sistemática de registros fotográficos, lo que constituyó una forma virtual de observación. Gracias a esta técnica de observación se pudieron identificar rasgos característicos de los CAP. La observación en campo sin duda hubiera portado mayores elementos acerca de los CAP, pero las condiciones de la pandemia por COVID 19 lo impidieron. Aun así, antes del inicio de la pandemia, se evidencio la dificultad de realizar las observaciones 
directamente en campo, lo que aporta evidencias sobre la dificultad del estudio de las operaciones de recicladores informales en mercados emergentes.

En este trabajo se muestra la importancia del uso de SIG para la representación y análisis de redes que operan a gran escala, como las redes de CAP en la zona de estudio. El SIG pudo usarse como en la mayoría la literatura de gestión de RSU, para el análisis de los aspectos técnicos operativos de las redes de CAP. Sin embargo, se utilizó SIG para explorar la relación entre localización de CAP y características socioeconómicas de los AGEB en que se localizan. El campo de la gestión de RSU puede enriquecerse incluyendo el uso de herramientas SIG para el análisis de los impactos económicos y sociales del reciclaje informal.

La propuesta de valor de los CAP es netamente logística, proveer de materiales de los RSU a la industria de la valorización. La fuente de ingresos primordial es la venta de material recuperado de los RSU. Su principal estrategia es la consolidación y explotación de la economía de escala. Sus asociaciones clave están con los pequeños recolectores quienes proveen a bajo costo los materiales que comercializan. Los CAP hacen un uso intensivo del espacio público para almacenar y hacer operaciones de clasificación, carga y descarga de RSU, lo que aligera su estructura de costos. El equipamiento necesario para operar es básico, siendo la mayor inversión necesaria la de vehículos de carga. Los CAP generan riqueza y empleo, sin embargo, el valor logístico que agregan las actividades de adquisición que se realizan en estos pudiera ser marginal si se consideran las externalidades negativas no cuantificadas observadas. Agregar valor a los materiales de los RSU a escala local en lugar de sólo transportarlos y almacenarlos podría incrementar el margen de utilidad, lo que permitiría internalizar costos que actualmente son cargados a la sociedad. Explorar alternativas de valorización de RSU en empresas locales es una línea de investigación futura de este trabajo.

Un mercado justo de materiales valorizables provenientes de los RSU se hace necesario para mejorar las condiciones de trabajo en los CAP en México. La importancia social, económica y ambiental de estas redes de CAP debe reconocerse e incluirse en las políticas públicas de basura cero. El concepto de basura cero y la implementación de la economía circular en México, y otros países, debe necesariamente integrar estas redes informales de CAP.

\section{Referencias Bibliográficas}

Apaydin, O. (2007). Route optimization for solid waste collection: Trabzon (Turkey) case study. Global NEST Journal, 9(1), 6-11.

Anguera, M. T., \& Mendo, A. H. (2013). La metodología observacional en el ámbito del deporte. E-balonmano. com: Revista de Ciencias del Deporte, 9(3), 135-160.

Çetinsaya Özkir, V., Efendigil, T., Demirel, T., Çetin Demirel, N., Deveci, M., \& Topçu, B. (2015). A three-stage methodology for initiating an effective management system for electronic waste in Turkey. Resources, Conservation and Recycling, 96, 61-70. https://doi.org/10.1016/j.resconrec.2015.01.008

Centro de Investigación para el Desarrollo A.C. (2015). Diagnóstico de la situación energética en el Distrito Federal: retos, pendientes y potencialidades. https://bit.ly/3j74aGt 
Bendul, J. C., Rosca, E., \& Pivovarova, D. (2017). Sustainable supply chain models for base of the pyramid. Journal of Cleaner Production, 162, S107-S120 https://doi.org/10.1016/j.jclepro.2016.11.001

Cassen, R. (1987). Our Common Future: Report of the World Commision on Environement and Development. International Affairs, 64(1), 176. https://doi.org/10.2307/2621529

Chang, K. T. (2016). Geographic information system. In Richardson, D. (Ed). International Encyclopedia of Geography: People, the Earth, Environment and Technology. https://doi.org/10.1002/9781118786352.wbieg0152

de Dios Ortúzar, J. (2012). Modelos de demanda de transporte. Ediciones UC.

Denscombe, M. (2014). The good research guide: for small-scale social research projects. McGraw-Hill Education.

Escalona Guerra, E. (2014). Daños a la salud por mala disposición de residuales sólidos y líquidos en Dili, Timor Leste. Revista Cubana de Higiene y Epidemiología, 52(2), 270-277.

Farahbakhsh, A., \& Forghani, M. A. (2019). Sustainable location and route planning with GIS for waste sorting centers, case study: Kerman, Iran. Waste Management and Research, 37(3), 287-300. https://doi.org/10.1177/0734242X18815950

Fei, F., Qu, L., Wen, Z., Xue, Y., \& Zhang, H. (2016). How to integrate the informal recycling system into municipal solid waste management in developing countries: Based on a China's case in Suzhou urban area. Resources, Conservation and Recycling, 110, 75-86. https://doi.org/10.1016/j.resconrec.2016.03.019

Gómez Maturano, J. (2019). Un modelo conceptual para evaluar la sustentabilidad de las operaciones de recolección y adquisición de RSU. Virtual PRO. Procesos Industriales. Ingenio Colombiano IngCo SAS. https://bit.ly/3xMM94y

Gutiérrez Gallego, J. C. (2015). Estructuración de un modelo de negocio basado en el aprovechamiento de residuos eléctricos y electrónicos. Universidad EAFIT. https://bit.ly/35KQ8CL

Global Reporting Initiative. (2015). Sustainability and Reporting Trends in 2025, Preparing for the Future. GRI. https://bit.ly/3gOR4fp

Hincapie, E. C. (2018). Viabilidad en la exportación de RAEE (residuos de aparatos eléctricos y electrónicos) generados en los centros de acopio de población vulnerable en MedellínColombia. Oportunidad de negocio. Revista En-contexto/ISSN: 2346-3279, 6(9), 107-118. https://bit.ly/3vShloS

Horvath, B., Mallinguh, E., \& Fogarassy, C. (2018). Designing business solutions for plastic waste management to enhance circular transitions in Kenya. Sustainability, 10(5), 1664. https://doi.org/10.3390/su10051664

Instituto Nacional de Estadística Geografía e Informática (2010). Censo de población y vivienda. Censo de población y vivienda. https://bit.ly/3h16bBj

Instituto Nacional de Estadística Geografía e Informática. (2018). Sistema de Clasificación Industrial de América del Norte, México. https://bit.ly/3j8mHSM

Instituto Nacional de Estadística Geografía e Informática. (2019). Directorio Estadístico Nacional de Unidades Económicas DENUE. https://bit.ly/3zSWTjx 
Instituto para Políticas de Transporte y Desarrollo México (2016). Programa para el Desarrollo Bajo en Emisiones de México. https://bit.ly/3wTO8E7

Jenks, George F. (1967): "The Data Model Concept in Statistical Mapping", in International Yearbook of Cartography, 7, International Cartographic Association, pp. 186-190

Karadimas Nikolaos, V., Papatzelou, K. y Loumos, V.G. (2007). Optimal solid waste collection routes identified by the ant colony system algorithm. Waste Management and Research, 25(2), 139-147. 10.1177/0734242X07071312

López Romo, H. (2013). Nivel Socioeconómico AMAI, Comité Niveles Socioeconómicos AMAI/Instituto de Investigaciones Sociales SC. https://bit.ly/2Sm6vlY

Ley general para la prevención y gestión integral de residuos. (2003). En Cámara de Diputados del Honorable Congreso de la Unión. Diario oficial de la federación. Ley federal.

Morillas, A. V., Pérez, M. V., Valdemar, R. M. E., Contreras, M. M., Islas, S. H., Guillén, M. Y. L. O., y Filgueira, H. J. A. (2016). Generación, legislación y valorización de residuos plásticos en Iberoamérica. Revista Internacional de Contaminación Ambiental, 32, 63-76. http://dx.doi.org/10.20937/RICA.2016.32.05.05

Parmigiani, A., \& Rivera-Santos, M. (2015). Sourcing for the base of the pyramid: Constructing supply chains to address voids in subsistence markets. Journal of Operations Management, 33-34(1), 60-70. https://doi.org/10.1016/j.jom.2014.10.007

Pires, A., Martinho, G., \& Chang, N.-B. (2011). Solid waste management in European countries: A review of systems analysis techniques. Journal of Environmental Management, 92(4), 1033-1050. https://doi.org/10.1016/j.jenvman.2010.11.024

Rosca, E., Arnold, M., y Bendul, J. C. (2017). Business models for sustainable innovation-an empirical analysis of frugal products and services. Journal of Cleaner Production, 162, S133-S145. https://doi.org/10.1016/j.jclepro.2016.02.050

Sanjeevi, V., \& Shahabudeen, P. (2016). Optimal routing for efficient municipal solid waste transportation by using ArcGIS application in Chennai, India. Waste Management \& Research, 34(1), 11-21. https://doi.org/10.1177/0734242X15607430

Secretaría del Medio Ambiente de la Ciudad de México (2016). Programa de Gestión Integral de Residuos Sólidos 2016-2020. https://bit.ly/35Ovl0Z

Secretaría de Medio Ambiente y Recursos Naturales (2016). Informe de la Situación del Medio Ambiente en México. Compendio de estadísticas ambientales, indicadores clave de desempeño ambiental y de crecimiento verde. Edición 2015. México. https://bit.ly/3gOqcMK

Strandhagen, J. O., Vallandingham, L. R., Fragapane, G., Strandhagen, J. W., Stangeland, A. B. H., \& Sharma, N. (2017). Logistics 4.0 and emerging sustainable business models. Advances in Manufacturing, 5(4), 359-369. https://doi.org/10.1007/s40436-0170198-1 